\title{
Overproduction of docosahexaenoic acid in Schizochytrium sp. through genetic engineering of oxidative stress defense pathways
}

\author{
Xiao Han, Zhaohui Li, Ying Wen and Zhi Chen *i)
}

\begin{abstract}
Background: Oxidation and peroxidation of lipids in microorganisms result in increased levels of intracellular reactive oxygen species (ROS) and reactive aldehydes, and consequent reduction of cell growth and lipid accumulation.

Results: To reduce oxygen-mediated cell damage and increase lipid and docosahexaenoic acid (DHA) production in Schizochytrium sp., we strengthened the oxidative stress defense pathways. Overexpression of the enzymes thioredoxin reductase (TRXR), aldehyde dehydrogenase (ALDH), glutathione peroxidase (GPO), and glucose-6-phosphate dehydrogenase (ZWF) strongly promoted cell growth, lipid yield, and DHA production. Coexpression of ZWF, ALDH, GPO, and TRXR enhanced ROS-scavenging ability. Highest values of dry cell weight, lipid yield, and DHA production $(50.5 \mathrm{~g} / \mathrm{L}, 33.1 \mathrm{~g} / \mathrm{L}$, and $13.3 \mathrm{~g} / \mathrm{L}$, respectively) were attained in engineered strain OaldH-gpo-trxR by shake flask fedbatch culture; these were increases of $18.5 \%, 80.9 \%$, and $114.5 \%$ relative to WT values.
\end{abstract}

Conclusions: Our findings demonstrate that engineering of oxidative stress defense pathways is an effective strategy for promoting cell robustness, lipid yield, and DHA production in Schizochytrium.

Keywords: Schizochytrium sp., Docosahexaenoic acid, Genetic engineering, Oxidative stress defense pathway

\section{Background}

Docosahexaenoic acid (DHA) has received increasing research attention during the past two decades because of its beneficial effects on human health. DHA has been shown to reduce cardiovascular disease risk, lower blood pressure, and exert various anti-inflammatory effects $[1,2]$. The unicellular marine eukaryote Schizochytrium (class Labyrinthulomycetes; family Thraustochytriaceae) is an algae-like microorganism utilized commercially for production of DHA-rich oil and (in dried form) as a source of DHA in animal feeds, human foods, and nutritional supplements [3-5]. Total fatty acids (TFAs)

*Correspondence: chenzhi@cau.edu.cn

State Key Laboratory of Agrobiotechnology, College of Biological

Sciences, China Agricultural University, Beijing 100193, China comprise up to $70 \%$ of cell weight of Schizochytrium sp., and $25-45 \%$ of TFAs are DHA [5, 6]. Traditional culture methods and genetic engineering techniques have been used by many research groups to increase DHA production by Schizochytrium sp. [7-12].

Oxygen supply plays a key role in cell proliferation and lipid accumulation of Schizochytrium sp. [13, 14]. High oxygen supply shortens fermentation period and increases dry cell weight (DCW). Lipids, particularly polyunsaturated fatty acids (PUFAs), are highly susceptible to free radical attack. Acyl-CoA oxidase catalyzes the first step of $\beta$-oxidation of fatty acyl-CoA and generates hydrogen peroxide [15], and hydroxyl radical $(\cdot \mathrm{OH})$ and oxygen induce oxidation of unsaturated fatty acids and generate lipid peroxide [16]. Autolysis of resulting lipid peroxide generates a variety of lipid-derived aldehydes 
and ketones $[17,18]$. Reactive oxygen species (ROS) and reactive aldehydes produced in this manner cause oxidation of proteins, lipids, and nucleic acids, with consequent disruption of DNA replication, loss of protein function, and even cell death $[19,20]$. Microbial cells utilize various detoxification enzymes and non-enzymatic defensive mechanisms to protect cellular components from ROS and reactive aldehydes [21]. Major antioxidant enzymes include catalase (CAT), superoxide dismutase (SOD), and glutathione peroxidase (GPO). Reduced glutathione (GSH) and thioredoxin (TRX) are important non-enzymatic small molecules that scavenge ROS in cells [22, 23]. Glutathione reductase (GSR) catalyzes NADPH-dependent reduction of glutathione disulfide (GSSG) to GSH [23], and thioredoxin reductase (TRXR) utilizes NADPH for reduction of active-site disulfide of TRX [16]. Aldehyde dehydrogenase (ALDH) is an oxidizing enzyme involved in detoxification of both exogenous and endogenous aldehydes [24].

During microbial fermentation for production of lipids and PUFAs, generated ROS and reactive aldehydes impair cell metabolism and suppress lipid productivity. It is therefore crucial to control levels of intracellular oxidative species. In a study of the marine microalga Crypthecodinium cohnii, Liu et al. [25] reduced intracellular ROS concentration by adding the antioxidant sesamol, which significantly increased biomass and DHA content. Ren et al. [3], by adding the antioxidant ascorbic acid $(9 \mathrm{~g} / \mathrm{L})$ to fermentation medium for Schizochytrium sp., obtained respective $16.16 \%$ and $30.44 \%$ increase in yields of DCW and DHA. Thus, reducing ROS levels of oleaginous microorganisms appears to be an effective strategy for enhancing cell robustness and DHA production. In studies of yeast, overexpression of ROS-scavenging enzymes reduced intracellular ROS, thereby promoting thermotolerance, robustness, and ethanol production [23, 26]. $\mathrm{Xu}$ et al. [16] produced an engineered Yarrowia lipolytica strain with high lipid titer $(72.7 \mathrm{~g} / \mathrm{L})$ and high lipid content (81.4\%) through combined overexpression of three enzymes (GSR, GPO, ALDH) of cellular oxidative stress defense pathways, and of glucose-6-phosphate dehydrogenase (ZWF), which generates NADPH for reduction of GSH and TRX disulfides.

In this study, we strengthened cellular oxidative stress defense pathways and aldehyde detoxification pathway in Schizochytrium sp. ATCC20888 through overexpression of several ROS-scavenging and aldehyde-scavenging enzymes, resulting in significant enhancement of cell growth, DHA production, and lipid production. In view of its robustness and high productivity, this engineered strain has strong potential as a Schizochytrium platform for efficient production of PUFAs and fatty acid-derived oleochemicals.

\section{Results and discussion}

Engineering oxidative stress defense pathways in Schizochytrium sp.

During aerobic fermentation, oxidation and peroxidation of lipids result in increased intracellular levels of ROS and reactive aldehydes, which affect cell growth and lipid accumulation [16]. We attempted to enhance the oxidative stress defense system of Schizochytrium to prevent or reduce oxidation of DHA and lipids. The key enzymes of the oxidative stress defense system for removal of oxygen free radicals and their products are GPO, GSR, SOD1, ALDH, and TRXR [22] (Fig. 1). GSR and TRXR both require NADPH as a reductant against oxidative stress. $\mathrm{ZWF}$ is an enzyme involved in the pentose phosphate

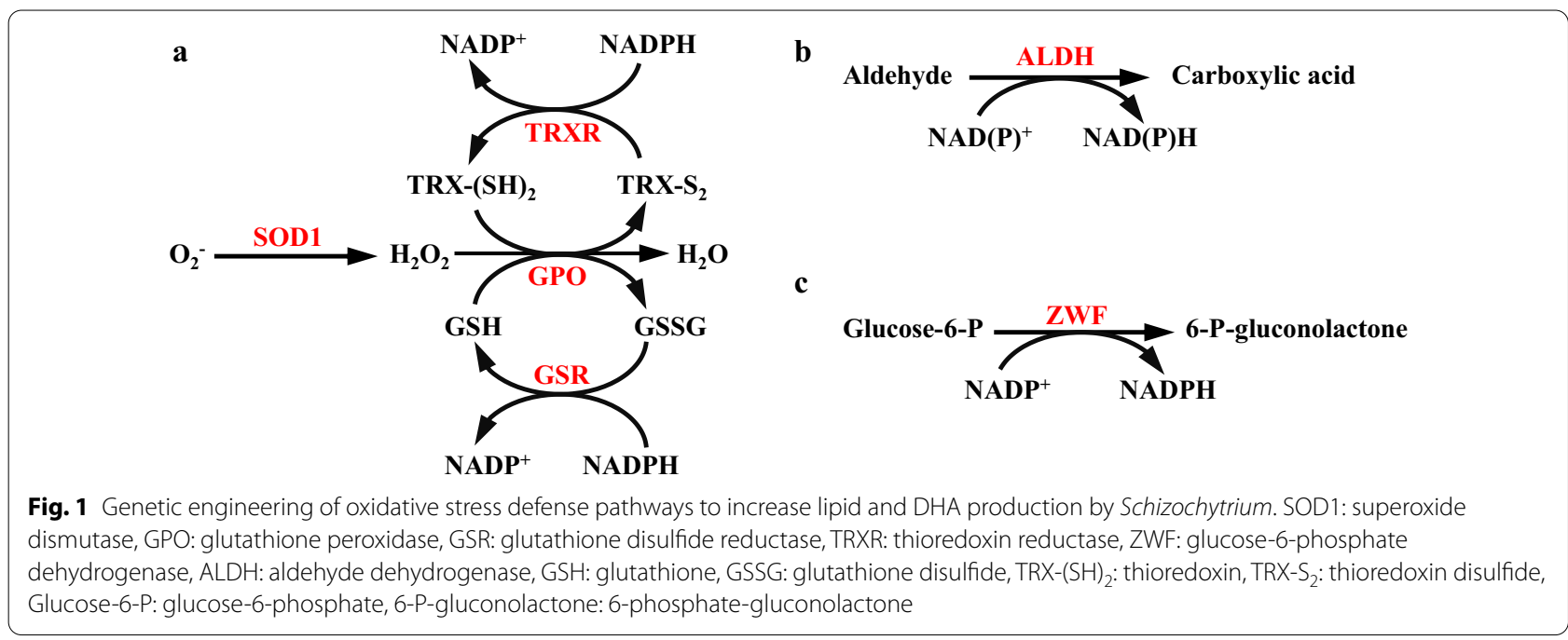


pathway, the primary pathway for production of NADPH by cells [23] (Fig. 1). We therefore expected that overexpression of these antioxidant enzymes in Schizochytrium would help maintain redox homeostasis, and thereby promote cell growth and production of lipids and DHA.

The genes that encode GPO (gpo; YALI0_E02310g), GSR (gsr; YALI0_E18029g), SOD1 (sod1; YALI0_ E12133g), and TRXR (trxR; YALI0_D27126g) were amplified from Y. lipolytica Po1f cDNA, and aldH (EG10036) was amplified from $E$. coli DH5 $\alpha$ genomic DNA. BLAST search of the Schizochytrium sp. CCTCC M209059 genome [27] revealed one putative glucose-6-phosphate dehydrogenase-encoding gene ( $z w f$; Additional file 2: Table S1). $z w f$ was amplified from cDNA of Schizochytrium sp. ATCC20888. The above genes were cloned separately into PPICZ $\alpha \mathrm{A}$ under $c c g 1$ promoter or TEF-1 promoter (Additional file 1: Figure S1), and corresponding transformants (Ogpo, Ogsr, Osod1, OtrxR, OaldH, Ozwf) were then obtained by electrotransformation.

\section{Overexpression of oxidative stress defense enzymes enhanced Schizochytrium sp. cell growth and lipid production}

Overexpression of antioxidant enzymes as above enhanced growth of Schizochytrium sp. ATCC20888 in shake-flask fermentation experiments (Fig. 2). DCW values for Ogsr, Osod1, Ozwf, OaldH, Ogpo, and OtrxR were increased $4.3-7.7 \%$ relative to WT value $(23.5 \mathrm{~g} / \mathrm{L})$. Lipid production of overexpression strains was also increased significantly. Relative to WT value (52.3\%), lipid content was much higher for OtrxR (63.3\%), Ogpo

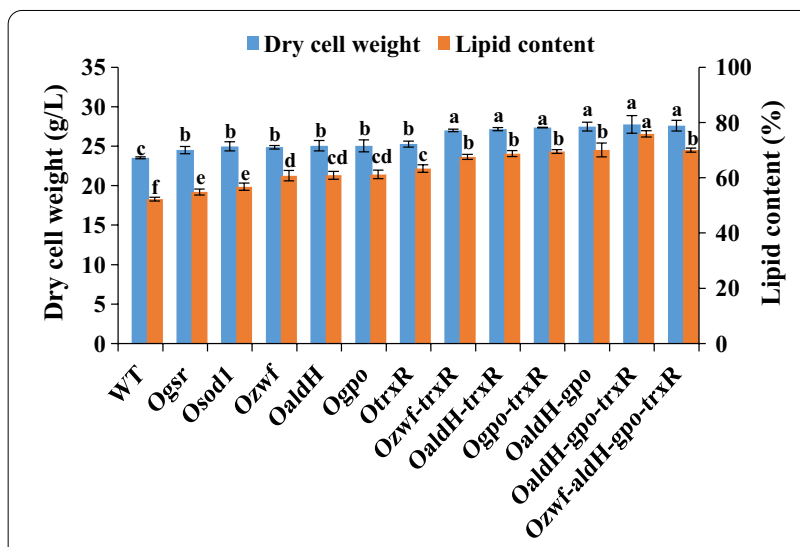

Fig. 2 Effects on cell growth and lipid accumulation by overexpression of oxidative stress defense enzymes in various Schizochytrium strains as described in M\&M. DCW ( $\mathrm{g} / \mathrm{L}$ ) and lipid content (\% DCW) are indicated on left and right Y-axes. Error bars: SD from three replicate experiments. Data were analyzed by one-way ANOVA and Duncan's multiple range test, using SPSS V. 23.0. Differing lowercase letters indicate significant difference $(p<0.05)$ between values
(61.2\%), OaldH (60.9\%), and Ozwf (60.8\%), and slightly higher for Ogsr (54.9\%) and Osod1 (56.7\%). Introduction of the control plasmid (pPICZ-ccg1p) had no effects on DCW, lipid accumulation, and DHA production of WT (Additional file 1: Figure S2). The results indicated that the increase of DCW, lipid production and DHA yields in the overexpression strains was due to overexpression of the oxidative stress defense genes. In yeast studies, enhancement of GSH and TRX antioxidant systems strongly promoted cell growth and production of ethanol and lipids $[16,23]$. In the present study, enzyme overexpression had a stronger promoting effect on Schizochytrium cell growth and lipid production for TRXR than for other enzymes (Fig. 2). In mammalian cells, ALDH plays the major role in prevention of lipotoxicity caused by free radical attack on lipids [28]. OaldH had much higher DCW and lipid content than did WT, even though the ald $H$ gene was from $E$. coli. ZWF catalyzed production of NADPH, which in addition to serving as reductant for GSR and TRXR also participates directly in fatty acid synthesis [29] - a process more conducive to lipid accumulation. Therefore, these findings demonstrate the essential roles of TRXR, ALDH, GPO, and ZWF in elimination of oxygen free radicals and reactive aldehydes in Schizochytrium.

We next attempted to further increase lipid accumulation by overexpressing ZWF, ALDH, GPO, and TRXR in combinations of two, three, or four. Lipid yields of OzwftrxR, OaldH-trxR, Ogpo-trxR, and OaldH-gpo were notably higher than those of (respectively) Ozwf, OaldH, Ogpo, and OtrxR (Fig. 2; Table 1). That is, yields were increased by $48.8 \%$ in Ozwf-trxR, $52.0 \%$ in OaldH-trxR, $54.5 \%$ in Ogpo-trxR, and $56.9 \%$ in OaldH-gpo, relative to WT value $(12.3 \mathrm{~g} / \mathrm{L})$ (Table 1$)$. Antioxidant function of GSH and TRX in the yeast Saccharomyces cerevisiae was promoted by increased NADPH supply in previous studies [23, 26], and we achieved a similar result in Schizochytrium by coupling TRXR with ZWF (Fig. 2). Maximal DCW (27.8 g/L) and lipid yield (21.0 g/L) were achieved in OaldH-gpo-trxR (co-overexpression of ALDH, GPO, and TRXR). These values were respectively $18.3 \%$ and $70.7 \%$ higher than WT values (Table 1 ). However, overexpression of $z w f$ in OaldH-gpo-trxR did not further improve DCW $(27.6 \mathrm{~g} / \mathrm{L})$ and lipid yield $(19.3 \mathrm{~g} / \mathrm{L})$ (Table 1; Additional file 1: Figure S2), and the possible reason might be that the simultaneous overexpression of the four genes increased the metabolic burden of Schizochytrium sp. RT-qPCR was performed to determine the transcription levels of $z w f$, aldH, gpo, and $\operatorname{trxR}$ genes in WT, WT/pPICZ-ccg1p, OaldH-gpo-trxR, and Ozwf-aldH-gpo-trxR. No significant difference in the transcription levels of the tested genes was observed between WT and the control strain WT/pPICZ-ccg1p, 
Table 1 Fermentation characteristics of overexpression strains of Schizochytrium sp.

\begin{tabular}{|c|c|c|c|c|}
\hline Strain & $\mathrm{DCW}(\mathrm{g} / \mathrm{L})$ & Lipid yield (g/L) & Lipid content (\%) & DHA yield ( $\mathrm{g} / \mathrm{L})$ \\
\hline WT & $23.5 \pm 0.1^{b}$ & $12.3 \pm 0.1^{d}$ & $52.3^{c}$ & $4.3 \pm 0.1^{d}$ \\
\hline Ozwf-trxR & $27.0 \pm 0.2^{\mathrm{a}}$ & $18.3 \pm 0.4^{c}$ & $67.6^{b}$ & $7.0 \pm 0.1^{c}$ \\
\hline $\begin{array}{l}\text { OaldH-trxR } \\
\text { Ogpo-trxR } \\
\text { OaldH-gpo } \\
\text { OaldH-gpo-trxR } \\
\text { Ozwf-aldH-gpo-trxR }\end{array}$ & $\begin{array}{l}27.2 \pm 0.1^{\mathrm{a}} \\
27.4 \pm 0.2^{\mathrm{a}} \\
27.5 \pm 0.6^{\mathrm{a}} \\
27.8 \pm 1.1^{\mathrm{a}} \\
27.6 \pm 0.6^{\mathrm{a}}\end{array}$ & $\begin{array}{l}18.7 \pm 0.2^{b c} \\
19.0 \pm 0.3^{b c} \\
19.3 \pm 0.8^{b} \\
21.0 \pm 0.4^{a} \\
19.3 \pm 0.8^{a}\end{array}$ & $\begin{array}{l}68.7^{b} \\
69.5^{b} \\
70.1^{b} \\
75.8^{a} \\
70.0^{b}\end{array}$ & $\begin{array}{l}7.1 \pm 0.1^{b c} \\
7.4 \pm 0.2^{b} \\
7.3 \pm 0.1^{b c} \\
8.8 \pm 0.3^{a} \\
8.4 \pm 0.2^{a}\end{array}$ \\
\hline
\end{tabular}

Data were analyzed by one-way ANOVA and Duncan's multiple range test, using SPSS V. 23.0. Differing lowercase letters indicate significant difference $(p<0.05)$ between values

while the transcription levels of aldH, gpo, and trxR were greatly increased in OaldH-gpo-trxR, and the expression of the four tested genes was increased in Ozwf-aldH-gpotrxR compared with WT (Fig. 3). In summary, coupling of oxidative stress defense pathways and aldehyde detoxification pathway in Schizochytrium notably promoted cell growth and reduced lipid oxidation.

\section{Overexpression of ALDH, GPO, TRXR, and ZWF increased DHA production}

To investigate the possibility of increasing DHA production by enhancing oxidative stress defense pathways, we used gas chromatography to measure DHA yields of WT, Ozwf-trxR, OaldH-trxR, Ogpo-trxR, OaldH-gpo, OaldH-gpo-trxR, and Ozwf-aldH-gpo-trxR. DHA production in the overexpression strains was significantly increased (Fig. 4a), similarly to results for lipid production. DHA yields of Ozwf-trxR (7.0 g/L), OaldH-trxR $(7.1 \mathrm{~g} / \mathrm{L})$, OaldH-gpo $(7.3 \mathrm{~g} / \mathrm{L})$, and Ogpo-trxR $(7.4 \mathrm{~g} / \mathrm{L})$ were $62.8-72.1 \%$ higher than that of WT $(4.3 \mathrm{~g} / \mathrm{L})$. The highest DHA yield observed was that of OaldH-gpotrxR $(8.8 \mathrm{~g} / \mathrm{L})$-- $104.7 \%$ higher than WT value. The DHA yield of Ozwf-aldH-gpo-trxR $(8.4 \mathrm{~g} / \mathrm{L})$ was slightly lower than that of OaldH-gpo-trxR (Fig. 4a; Table 1).
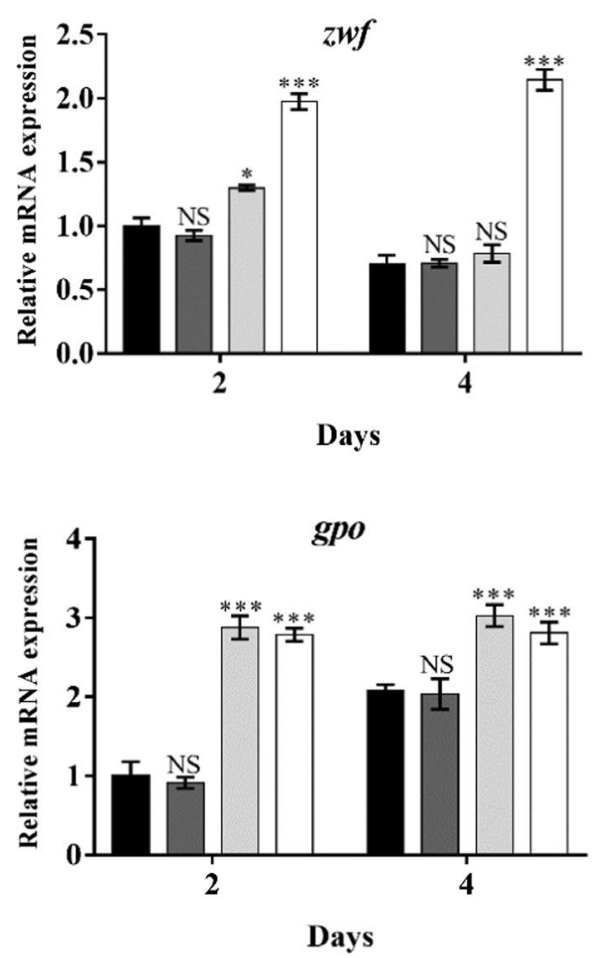

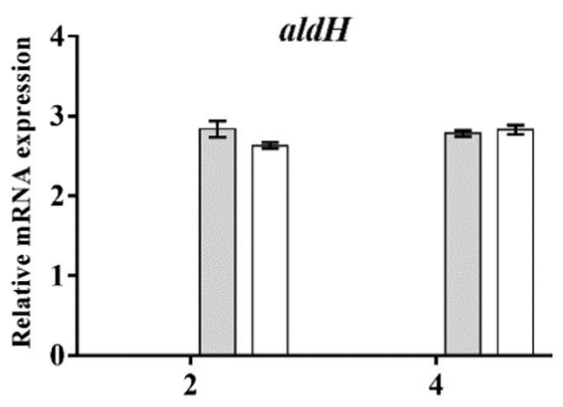

Days

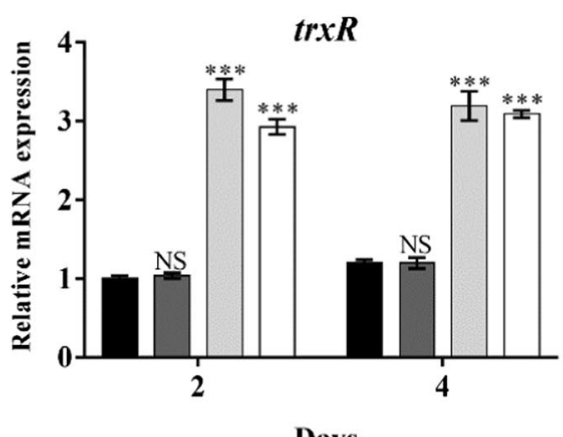

Fig. 3 qRT-PCR analysis of the transcription levels of the $z w f$, aldH, gpo, and trxR genes in WT, WT/pPICZ-ccg1p, OaldH-gpo-trxR, and Ozwf-aldH-gpo-trxR. RNAs were isolated from cells grown in fermentation broth for 2 and 4 days. ${ }^{* *}, p<0.001 ;{ }^{*}, p<0.05 ;$ NS, not significant by Student's t-test 


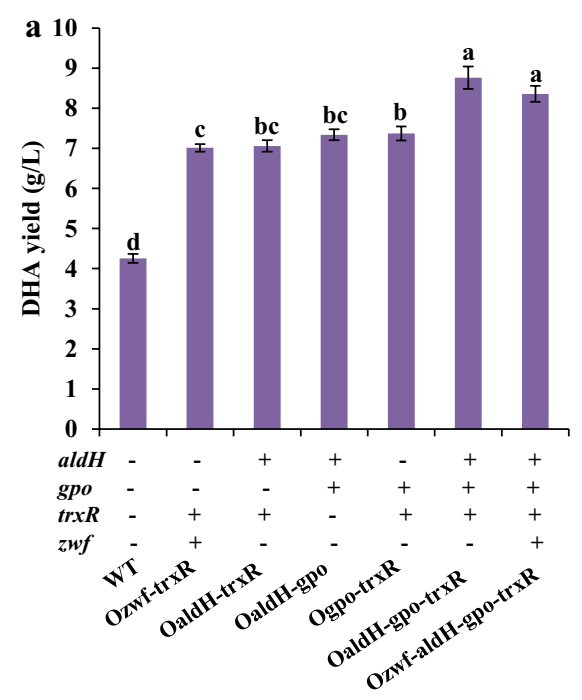

c

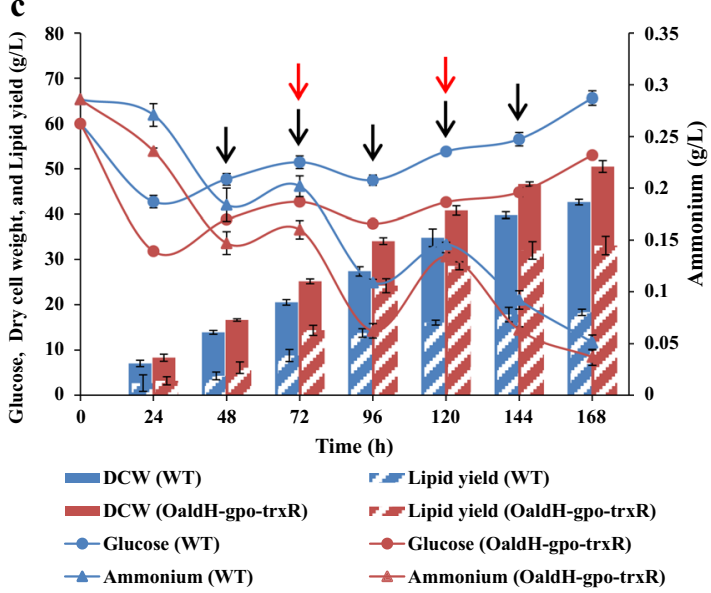

b
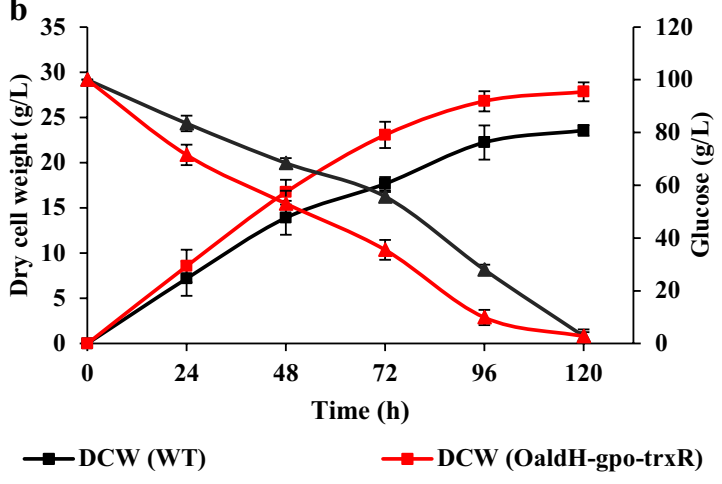

$\rightarrow$ DCW (OaldH-gpo-trxR) $\rightarrow$ Glucose (OaldH-gpo-trxR)

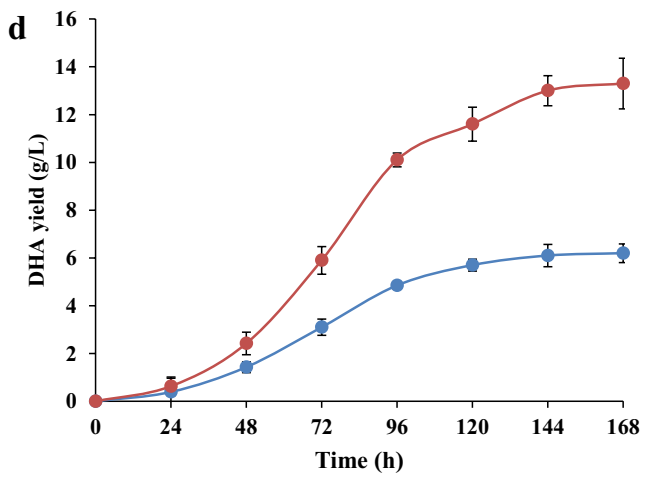

$\rightarrow$ DHA (WT) $\rightarrow$-DHA (OaldH-gpo-trxR)

Fig. 4 Effects of ALDH, GPO, TRXR, and ZWF overexpression on DHA production, cell growth, and carbon and nitrogen sources utilization. a DHA yield ( $\mathrm{g} / \mathrm{L}$ ). Cells were cultured in fermentation medium for $120 \mathrm{~h}$. b Growth curves and glucose consumption by WT and OaldH-gpo-trxR. $\mathbf{c}$, d WT and OaldH-gpo-trxR strains were cultured for $168 \mathrm{~h}$ in shake flask fed-batch fermentation. The fermentation broth was collected every $24 \mathrm{~h}$ to determine the concentrations of glucose and ammonium, DCW, lipid and DHA yields. Black arrow: $25 \mathrm{~g} / \mathrm{L}$ glucose was supplied to the culture every $24 \mathrm{~h}$ from 48 to $144 \mathrm{~h}$. Red arrow: $2 \mathrm{~g} / \mathrm{L}$ yeast extract was supplied to the fermentation culture at $72 \mathrm{~h}$ and $120 \mathrm{~h}$, respectively

Therefore, OaldH-gpo-trxR was used for further analysis. The growth curves and glucose consumption curves of WT and OaldH-gpo-trxR showed striking differences. OaldH-gpo-trxR had faster growth rate, much faster glucose consumption rate, and higher DCW than WT (Fig. 4b). These findings demonstrate that overexpression of ALDH, GPO, and TRXR promoted robustness, carbon source uptake, and growth of Schizochytrium. Next, we performed fed-batch fermentation to test the ability of OaldH-gpo-trxR to produce lipids and DHA, together with the control strain. The initial concentrations of glucose and yeast extract were $60 \mathrm{~g} / \mathrm{L}$ and $3 \mathrm{~g} / \mathrm{L}$, and then $125 \mathrm{~g}$ glucose and $4 \mathrm{~g}$ yeast extract were added in 5 and 2 times after $48 \mathrm{~h}$ and $72 \mathrm{~h}$, respectively. The carbon and nitrogen sources consumption rates of OaldH-gpo-trxR were still much faster than those of WT (Fig. 4c). In both strains, the output of DCW, lipid and DHA increased rapidly from 48 to $96 \mathrm{~h}$, and the synthesis of lipid and DHA became stable after $120 \mathrm{~h}$ (Fig. 4c, d). The DCW and the yields of lipid and DHA in OaldH-gpo-trxR reached 50.5, 33.1, and $13.3 \mathrm{~g} / \mathrm{L}$ after $168 \mathrm{~h}$ of fermentation with $185 \mathrm{~g} / \mathrm{L}$ glucose, which were increased by $18.5 \%, 80.9 \%$, and $114.5 \%$ compared with WT $(42.6,18.3$, and $6.2 \mathrm{~g} / \mathrm{L}$ ).

Various strategies have been used to increase DHA production of Schizochytrium, including traditional fermentation conditions optimization and metabolic engineering (Table 2). Chen et al. [30] increased DHA 
Table 2 DHA yield in Schizochytrium sp. from the literatures and this study

\begin{tabular}{|c|c|c|c|c|c|}
\hline Schizochytrium sp. & Strategy & Cultivation mode & Yield (g/L) & Content (\%TFA) & Reference \\
\hline $\begin{array}{l}\text { S056 } \\
\text { HX-308 }\end{array}$ & $\begin{array}{l}\text { Optimization of sea salt } \\
\text { ALE }\end{array}$ & $\begin{array}{l}\text { Shake flask } \\
\text { 1,500-L fermentor }\end{array}$ & $\begin{array}{l}8.0 \\
38.1\end{array}$ & $\begin{array}{l}44.4 \\
53.3\end{array}$ & $\begin{array}{l}{[30]} \\
{[31]}\end{array}$ \\
\hline$H X-308$ & Addition of ascorbic acid & 5-L bioreactor & 38.3 & 54.5 & {$[3]$} \\
\hline SR21 & Addition of inositol & Shake flask & 8.5 & 37.3 & [33] \\
\hline $\begin{array}{l}\text { HX-308 } \\
\text { MYA1381 } \\
\text { ATCC20888 } \\
\text { ATCC20888 } \\
\text { ATCC20888 }\end{array}$ & $\begin{array}{l}\text { Continuous feeding } \\
\text { Metabolic engineering } \\
\text { Metabolic engineering } \\
\text { Metabolic engineering } \\
\text { Metabolic engineering }\end{array}$ & $\begin{array}{l}\text { Fed-batch } \\
\text { Shake-flask/Fed-batch } \\
\text { Shake-flask } \\
\text { Shake-flask } \\
\text { Shake-flask/ Fed-batch }\end{array}$ & $\begin{array}{l}38.4 \\
7.0 / 47.4 \\
3.5 \\
6.4 \\
8.8 / 13.3\end{array}$ & $\begin{array}{l}55.0 \\
42.9 \\
\text { N.C } \\
37.9 \\
42.8\end{array}$ & $\begin{array}{l}{[32]} \\
{[11]} \\
{[12]} \\
{[10]} \\
\text { This study }\end{array}$ \\
\hline
\end{tabular}

ALE: Adaptive laboratory evolution, N.C.: not calculated

production of Schizochytrium sp. S056 through optimization of sea salt in the culture medium. Sun et al. [31] and Guo et al. [32] used adaptive laboratory evolution method and continuous feeding strategy to achieve DHA production of $38.1 \mathrm{~g} / \mathrm{L}$ and $38.4 \mathrm{~g} / \mathrm{L}$ in Schizochytrium sp. HX-308, respectively. The addition of inositol in the medium increased the lipid and DHA production of Schizochytrium sp. SR21 [33]. Recent studies also indicated that metabolic engineering is an efficient strategy to enhance lipid and DHA production of Schizochytrium. Wang et al. [12] increased lipid content and the proportion of odd fatty acids in Schizochytrium sp. S31 through increasing the intracellular NADPH supply and the acetyl-CoA carboxylase activity. Li et al. [11] overexpressed the malonyl CoA: ACP transacylase in Schizochytrium sp. MYA1381 and increased the DHA yield to $7.0 \mathrm{~g} / \mathrm{L}$ in shake-flask fermentation and to $47.4 \mathrm{~g} / \mathrm{L}$ in fed-batch fermentation. Here, we increased the DHA yield to $13.3 \mathrm{~g} / \mathrm{L}$ in shake flask fed-batch fermentation through enhancing the oxidative stress defense pathways in Schizochytrium sp. ATCC20888 (Table 2). To our best knowledge, the DHA yield obtained by OaldH-gpo-trxR is at a high level in the shake-flask fermentation of Schizochytrium sp.

Overexpression of ALDH, GPO, and TRXR affected fatty acid composition as well as increasing DHA yield. Fatty acid composition analysis revealed that, relative to WT values, the percentage of TFAs corresponding to major saturated fatty acids (myristic acid [C14:0] and palmitic acid [C16:0]) in OaldH-gpo-trxR was reduced, percentage for docosapentaenoic acid (DPA, C22:5) was slightly increased, and percentage for DHA was significantly increased (42.8\%; WT value was 37.7\%) (Fig. 5). In a previous study, PUFAs were readily oxidized for protection of cells from oxidative damage. Addition of omega-3 fatty acids to human cells reduced intracellular ROS formation, suggesting that these molecules may function as antioxidants [34]. During aerobic fermentation process in our study, PUFAs (DHA and

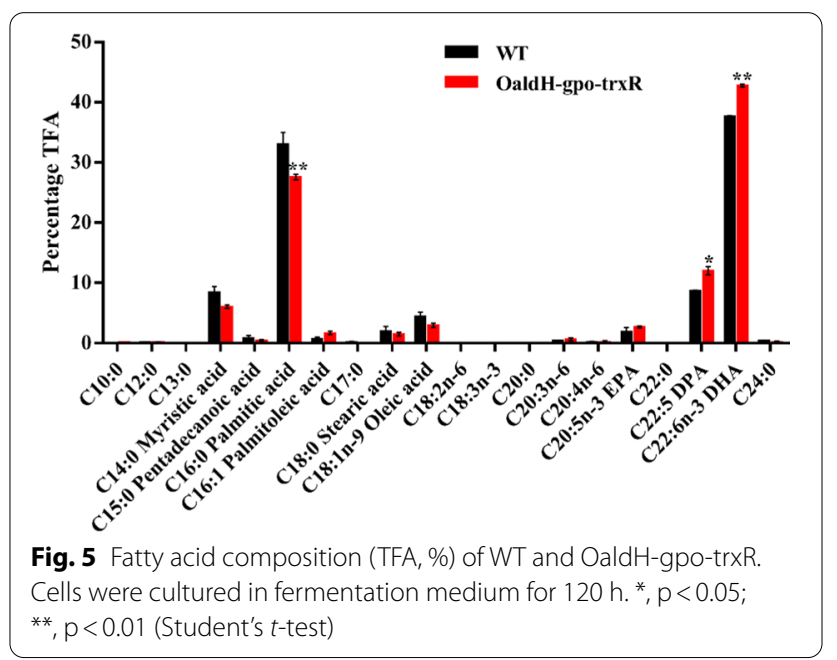

DPA) in TFAs of Schizochytrium were more readily oxidized than were saturated fatty acids for protection of cells from ROS damage, and maintenance of intracellular redox homeostasis. DHA production in both Schizochytrium sp. and Crypthecodinium cohnii $[3,25]$ was enhanced by addition of antioxidants. We also observed that enhancement of oxidative stress defense pathways in Schizochytrium increased production of PUFAs (particularly DHA).

\section{Overexpression of ALDH, GPO, and TRXR reduced intracellular ROS levels}

To examine the possibility that enhanced oxidative stress defense ability was the cause of increased growth rate and lipid accumulation in our overexpression strains, we measured intracellular ROS levels in WT and OaldHgpo-trxR during fermentation process. In both strains, ROS levels declined gradually at the beginning of fermentation, reached minimal values at $48 \mathrm{~h}$, and subsequently showed rapid increase (Fig. 6a); that is, intracellular ROS increased rapidly during lipid synthesis/ accumulation stage. OaldH-gpo-trxR, relative to WT, showed much 

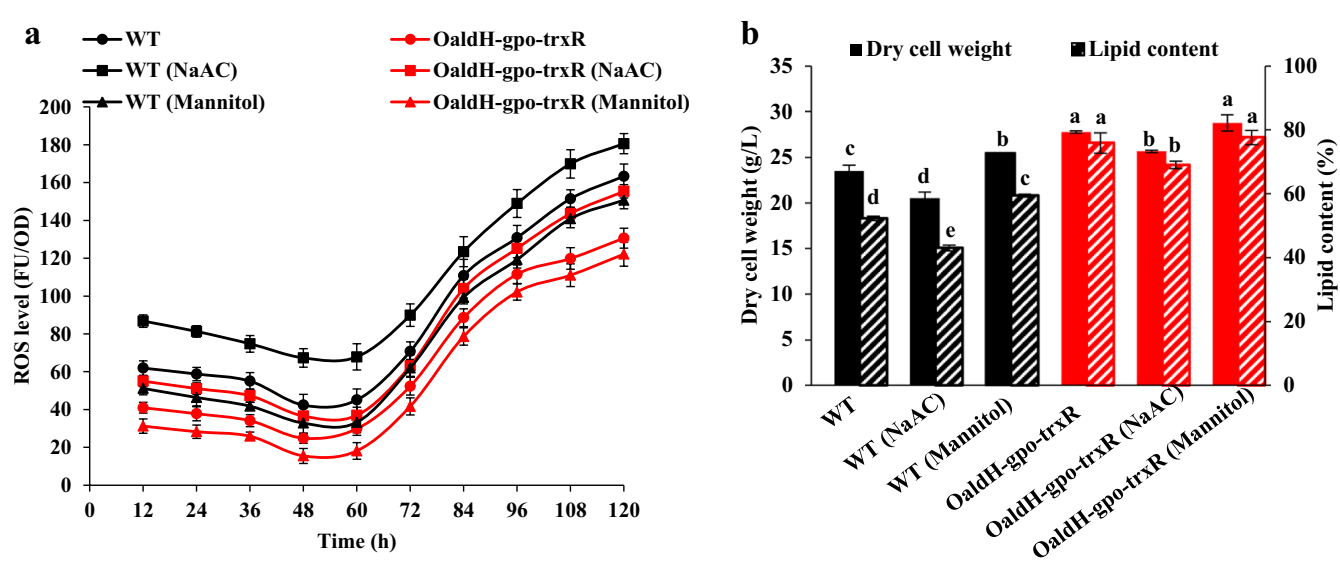

Fig. 6 Effects of ALDH, GPO, and TRXR overexpression on ROS levels in WT and OaldH-gpo-trXR. a ROS level (FU/OD 600$)$. b DCW (g/L) and lipid content (\% DCW). Cells were cultured for $120 \mathrm{~h}$ in fermentation medium with or without $0.3 \mathrm{M} \mathrm{NaOAc}$ or $60 \mathrm{mM}$ mannitol

lower ROS level but much higher DCW and lipid yield (Fig. 6b). In previous studies, weak acids, e.g., acetic acid, were found to promote ROS accumulation and induce oxidative stress [35, 36]. Mannitol scavenged hydroxyl radicals and protected cells from oxidative stress [37]. We therefore added $0.3 \mathrm{M}$ sodium acetate $(\mathrm{NaOAc})$ or $60 \mathrm{mM}$ mannitol to fermentation medium to evaluate the ROSscavenging ability of OaldH-gpo-trxR. In WT, NaOAc addition resulted in increase of ROS level from 163.3 to $180.6 \mathrm{FU} / \mathrm{OD}_{600}$ at $120 \mathrm{~h}$, and reductions of $\mathrm{DCW}$ and lipid yield by $12.8 \%$ and $28.5 \%$, respectively (Fig. 6). In OaldH-gpo-trxR, NaOAc addition also increased ROS level and reduced DCW and lipid content, indicating that cell growth and lipid accumulation were inhibited by increased ROS. In NaOAc-treated OaldH-gpo-trxR, relative to untreated WT, ROS level was lower (Fig. 6a), and DCW and lipid content were higher (Fig. 6b). Addition of mannitol resulted in reduced ROS level and increased DCW and lipid content for both WT and OaldH-gpotrxR. ROS level of mannitol-treated WT was higher than that of untreated OaldH-gpo-trxR (Fig. 6a). These findings, taken together, indicate that ROS-scavenging ability of Schizochytrium was notably increased by overexpression of ZWF, ALDH, GPO, and TRXR. Evidently, enhancement of oxidative stress defense pathways and aldehyde detoxification pathway resulted in detoxification of intracellular ROS and lipid-derived aldehydes, prevented reactive radicals from attacking nucleophilic centers of bioactive molecules, and thereby improved cell robustness and lipid production.

\section{Conclusions}

We overexpressed six ROS-scavenging and aldehydescavenging enzymes in Schizochytrium sp. for the purpose of improving cell robustness and DHA production.
Overexpression of ZWF, ALDH, GPO, and TRXR strongly promoted cell growth, lipid yield, and DHA production. We generated a DHA high-producer, OaldHgpo-trxR, that attained $50.5 \mathrm{~g} / \mathrm{L}$ DCW, $33.1 \mathrm{~g} / \mathrm{L}$ lipid yield, and $13.3 \mathrm{~g} / \mathrm{L}$ DHA production in shake flask fedbatch fermentation, respectively, $18.5,80.9$, and $114.5 \%$ increases relative to WT values. Our findings illustrate a new method for ROS reduction in Schizochytrium sp., and provide an effective strategy for generation of DHA high-producing strains.

\section{Methods}

\section{Microorganisms and culture conditions}

Schizochytrium sp. ATCC20888, used as wild-type strain $(\mathrm{WT})$, was preserved in $20 \%(\mathrm{v} / \mathrm{v})$ glycerol at $-80^{\circ} \mathrm{C}$. Strains used in the study are listed in Table 3. E. coli was cultured in LB broth or agar, added when necessary with $50 \mu \mathrm{g} / \mathrm{mL}$ zeocin. Schizochytrium sp. were cultured under conditions and media as in our previous study [10]. All fermentation experiments were performed in triplicate, and results from a representative batch are presented. For ROS analysis, cells were cultured for $120 \mathrm{~h}$ in fermentation medium supplemented with $0.3 \mathrm{M}$ sodium acetate or $60 \mathrm{mM}$ mannitol, on a rotary shaker $(250 \mathrm{rpm})$.

The fed-batch fermentation was carried out in the shake flasks and started with an initial glucose level of $60 \mathrm{~g} / \mathrm{L}$ and a yeast extract concentration of $3 \mathrm{~g} / \mathrm{L}$. The carbon source supplementation strategy is to supply $25 \mathrm{~g} / \mathrm{L}$ glucose to the culture every $24 \mathrm{~h}$ from 48 to $144 \mathrm{~h}$. The nitrogen source supplementation strategy is to supply $2 \mathrm{~g} / \mathrm{L}$ yeast extract to the fermentation culture at $72 \mathrm{~h}$ and $120 \mathrm{~h}$, respectively. The fermentation broth was collected every $24 \mathrm{~h}$ to determine the concentrations of glucose and ammonium, DCW, lipid and DHA yields. 
Table 3 Strains used in this study

\begin{tabular}{|c|c|c|}
\hline Strain & Description & Source or reference \\
\hline \multicolumn{3}{|l|}{ Schizochytrium sp. } \\
\hline ATCC 20888 & wild-type strain (WT) & $\begin{array}{l}\text { American Type Cul- } \\
\text { ture Collection }\end{array}$ \\
\hline WT/pPICZ-ccg1p & WT carrying control plasmid pPICZ-ccg1p & This study \\
\hline Ogsr & gsr overexpression strain & This study \\
\hline Osod1 & sod 1 overexpression strain & This study \\
\hline Ozwf & zwf overexpression strain & This study \\
\hline OaldH & aldH overexpression strain & This study \\
\hline Ogpo & gpo overexpression strain & This study \\
\hline OtrxR & $\operatorname{tr} x R$ overexpression strain & This study \\
\hline Ozwf-trxR & $z w f$ and trxR co-overexpression strain & This study \\
\hline OaldH-trxR & aldH and trxR co-overexpression strain & This study \\
\hline Ogpo-trxR & gpo and trxR co-overexpression strain & This study \\
\hline OaldH-gpo & aldH and gpo co-overexpression strain & This study \\
\hline OaldH-gpo-trxR & aldH, gpo, and trxR co-overexpression strain & This study \\
\hline Ozwf-aldH-gpo-trxR & $z W f$, aldH, gpo, and trxR co-overexpression strain & This study \\
\hline \multicolumn{3}{|l|}{ E. coli } \\
\hline JM109 & General cloning host for plasmid manipulation & Laboratory stock \\
\hline
\end{tabular}

\section{Plasmid construction}

For construction of oxidative stress defense pathwaysrelated plasmids, ald $H$ was amplified from $E$. coli $\mathrm{DH} 5 \alpha$ genomic DNA, gpo, gsr, sod1, and trxR were amplified from $Y$. lipolytica Po1f cDNA, and $z w f$ was amplified from Schizochytrium sp. ATCC20888 cDNA. aldH, trxR, and $z w f$ were promoted by $c c g 1$ promoter and terminator amplified from Neurospora expression vector pCCG.N$3 \mathrm{xMyc}$ [38], and gpo, gsr, and sod1were promoted by TEF-1 promoter and $C Y C-1$ terminator amplified from yeast expression vector pPICZ $\alpha$ A [39]. Primer pairs used in the study are listed in Additional file 2: Table S2. EcoRI/KpnI-digested promoter, KpnI/NotI-digested gene, and $N o t \mathrm{I} / \mathrm{XbaI}$-digested terminator were ligated simultaneously into EcoRI/XbaI-digested pPICZ $\alpha$ A to generate single gene overexpression plasmid (Additional file 1: Figure S1). Ligation reactions were performed overnight at $16^{\circ} \mathrm{C}$ using T4 DNA Ligase (TaKaRa Bio; Shiga, Japan). For overexpression of two, three, or four genes, promoter-gene-terminator expression cassette was amplified from single gene overexpression plasmid and inserted into XbaI-digested corresponding overexpression plasmid using Seamless Cloning and Assembly Kit (Clone Smarter Technologies; Houston, TX, USA). pPICZ $\alpha$ Accg1p-lacZ [10] was digested by KpnI/NotI to produce the control plasmid pPICZ-ccg1p.

\section{Strain construction}

PmeI-linearized overexpression plasmids were transformed into Schizochytrium competent cells to generate corresponding overexpression strains. Transformation of Schizochytrium was performed as described previously [40] with some modifications. In brief, Schizochytrium cells were cultured in seed medium for $24 \mathrm{~h}$, and were harvested by centrifugation $\left(5900 \mathrm{~g}, 4^{\circ} \mathrm{C}, 5 \mathrm{~min}\right)$ (HITACHI; Tokyo, Japan), washed with ice-cold sterile water and $1 \mathrm{M}$ sorbitol, and resuspended in $1 \mathrm{M}$ sorbitol. Plasmids were linearized by PmeI before electroporation. Linearized plasmid DNA $(5-10 \mu \mathrm{g})$ and competent cells were placed in a $0.1-\mathrm{cm}$-gap cuvette for electroporation $(1.5 \mathrm{kV}, 200 \Omega, 50 \mu \mathrm{F}$, twice). Cells were then added with $1 \mathrm{~mL}$ seed medium, incubated $4 \mathrm{~h}$ at $28^{\circ} \mathrm{C}$, spread on glucose/ peptone/ yeast extract (GPY) plates with $40 \mu \mathrm{g} / \mathrm{mL}$ zeocin, and grown at $28^{\circ} \mathrm{C}$ for selection of transformants.

\section{Analytical methods}

For determination of DCW, pellets from $40 \mathrm{~mL}$ fermentation broth were freeze-dried $(24-48 \mathrm{~h})$ to constant weight. Glucose concentration was determined by 3,5-dinitrosalicylic acid (DNS) method [41]. Total lipid content and fatty acid composition were analyzed as described previously [42, 43]. For lipid extraction, $\sim 0.3 \mathrm{~g}$ freeze-dried pellet was mixed with $6 \mathrm{~mL}$ of $4 \mathrm{M} \mathrm{HCl}$ for $30 \mathrm{~min}$, incubated in boiling water for $8 \mathrm{~min}$, added with $16 \mathrm{~mL}$ methanol/chloroform $(1: 1, \mathrm{v} / \mathrm{v})$, mixed vigorously, and centrifuged. The lower phase was transferred to a tube and evaporated 
under gentle nitrogen stream. Fatty acid methyl esters (FAMEs) were prepared from $30 \mathrm{mg}$ lipid sample and analyzed by gas chromatography (model GC522, Shanghai Wufeng Scientific Instruments Co.) with J\&W DB23 capillary column $(30 \mathrm{~m} \times 0.25 \mathrm{~mm}$ i.d.) (Agilent Technologies; Santa Clara, CA, USA), using nitrogen as carrier gas, injector temperature $250^{\circ} \mathrm{C}$, and injection volume $1 \mu \mathrm{L}$. Column temperature was raised from 150 to $200^{\circ} \mathrm{C}$ at $5^{\circ} \mathrm{C}$ per min, kept at $200^{\circ} \mathrm{C}$ for $1 \mathrm{~min}$, further raised to $230^{\circ} \mathrm{C}$ at $4^{\circ} \mathrm{C}$ per min, and kept at $230^{\circ} \mathrm{C}$ for 9 min.

\section{Quantitative real-time PCR (qRT-PCR) analysis}

Total RNAs were extracted from Schizochytrium sp. cells using TRIzol reagent (Tiangen; China) according to the manufacturer's protocol. The cDNA was prepared with M-MLV (RNase $\mathrm{H}^{-}$; TaKaRa Bio; Shiga, Japan) using oligo-dT18 from $4 \mu \mathrm{g}$ of total RNAs. qRT-PCR was performed as described previously [10]. The primers used for qRT-PCR are listed in Additional file 2: Table S2. The relative expression levels were determined according to the comparative $\mathrm{Ct}$ method, using actin as the internal control. Since aldH, gpo and trxR were from E. coli and $Y$. lipolytica, the transcriptional levels of the endogenous genes were analyzed in WT and WT/pPICZ-ccglp (Schizochytrium sp. does not contain the aldH gene), and of the transformed genes were analyzed in OaldH-gpotrxR and Ozwf-aldH-gpo-trxR.

\section{Determination of intracellular ROS levels}

These levels were determined using an ROS assay kit (Beijing Solarbio Science \& Technology Co.; China) as per manufacturer's instructions. Schizochytrium cells were suspended in $10 \mu \mathrm{M}$ DCFH-DA, incubated for $20 \mathrm{~min}$ at $37^{\circ} \mathrm{C}$ with occasional gentle stirring, and washed $3 \times$ with PBS to remove unincorporated DCFHDA. Fluorescence intensity was measured using a multifunctional plate reader (Molecular Devices; San Jose, CA, USA) with excitation and emission wavelengths $488 \mathrm{~nm}$ and $525 \mathrm{~nm}$, respectively.

\footnotetext{
Abbreviations

ALDH: Aldehyde dehydrogenase; CAT: Catalase; DCW: Dry cell weight; DHA: Docosahexaenoic acid; DNS: 3,5-Dinitrosalicylic acid; DPA: Docosapentaenoic acid; FAMEs: Fatty acid methyl esters; GPO: Glutathione peroxidase; GSH: Glutathione; GSR: Glutathione reductase; GSSG: Glutathione disulfide; NaOAc: Sodium acetate; PBS: Phosphate buffer saline; PUFAs: Polyunsaturated fatty acids; ROS: Reactive oxygen species; SOD1: Superoxide dismutase; TFAs: Total fatty acids; TRX: Thioredoxin; TRXR: Thioredoxin reductase; WT: Wild type; ZWF: Glucose-6-phosphate dehydrogenase.
}

\section{Supplementary Information}

The online version contains supplementary material available at https://doi. org/10.1186/s13068-021-01918-w.

Additional file 1: Figure S1. Schematic illustration of genetic constructs used for genomic integration. Figure S2. Effects of ZWF, ALDH, GPO, and TRXR overexpression on cell growth, lipid accumulation and DHA production. a DCW (g/L). b Lipid content (\% DCW). c DHA yield (g/L). Cells were cultured in fermentation medium for 1 to 5 days.

Additional file 2: Table S1. The open reading frame of zwf gene in Schizochytrium sp. Table S2. Primers used in this study.

\section{Acknowledgements \\ The authors are grateful to Dr. S. Anderson for English editing of the manuscript.}

\section{Authors' contributions}

$\mathrm{ZC}$ and $\mathrm{XH}$ designed the study. $\mathrm{XH}$ and $\mathrm{ZHL}$ performed the experiments. YW helped with analysis and discussion of results. $\mathrm{XH}$ and ZC wrote the manuscript. All authors read and approved the final manuscript.

\section{Funding}

This research did not receive any specific grant from funding agencies in the public, commercial, or not-for-profit sectors.

\section{Availability of supporting data}

All data supporting the conclusions of this article are included in the manuscript and in the additional information.

\section{Declarations}

Ethics approval and consent to participate

Not applicable.

\section{Consent for publication}

All authors have read and approved the final manuscript.

Competing interests

The authors declare that they have no competing interests.

Received: 29 October 2020 Accepted: 3 March 2021

Published online: 16 March 2021

\section{References}

1. Innes JK, Calder PC. The differential effects of eicosapentaenoic acid and docosahexaenoic acid on cardiometabolic risk factors: a systematic review. Int J Mol Sci. 2018;19:532.

2. Zarate R, El Jaber-Vazdekis N, Tejera N, Perez JA, Rodriguez C. Significance of long chain polyunsaturated fatty acids in human health. Clin Transl Med. 2017;6:25

3. Ren LJ, Sun XM, Ji XJ, Chen SL, Guo DS, Huang H. Enhancement of docosahexaenoic acid synthesis by manipulation of antioxidant capacity and prevention of oxidative damage in Schizochytrium sp. Bioresour Technol. 2017;223:141-8.

4. Barclay WR, Meager KM, Abril JR. Heterotrophic production of long-chain omega-3-fatty-acids utilizing algae and algae-like microorganisms. J Appl Phycol. 1994;6:123-9.

5. Ashford A, Barclay WR, Weaver CA, Giddings TH, Zeller S. Electron microscopy may reveal structure of docosahexaenoic acid-rich oil within Schizochytrium sp. Lipids. 2000;35:1377-86.

6. Raghukumar S. Thraustochytrid marine protists: production of PUFAs and other emerging technologies. Mar Biotechnol. 2008;10:631-40.

7. Chi ZY, Liu Y, Frear C, Chen SL. Study of a two-stage growth of DHAproducing marine algae Schizochytrium limacinum SR21 with shifting dissolved oxygen level. Appl Microbiol Biotechnol. 2009;81:1141-8. 
8. Ganuza E, Anderson AJ, Ratledge C. High-cell-density cultivation of Schizochytrium sp in an ammonium/pH-auxostat fed-batch system. Biotechnol Lett. 2008;30:1559-64.

9. Li Z, Ling X, Zhou H, Meng T, Zeng J, Hang W, et al. Screening chemical modulators of benzoic acid derivatives to improve lipid accumulation in Schizochytrium limacinum SR21 with metabolomics analysis. Biotechnol Biofuels. 2019;12:209.

10. Han X, Zhao Z, Wen Y, Chen Z. Enhancement of docosahexaenoic acid production by overexpression of ATP-citrate lyase and acetyl-CoA carboxylase in Schizochytrium sp. Biotechnol Biofuels. 2020;13:131.

11. Li Z, Meng T, Ling X, Li J, Zheng C, Shi Y, et al. Overexpression of malonylCoA: ACP transacylase in Schizochytrium sp. to improve polyunsaturated fatty acid production. J Agric Food Chem. 2018;66:5382-91.

12. Wang F, Bi Y, Diao J, Lv M, Cui J, Chen L, et al. Metabolic engineering to enhance biosynthesis of both docosahexaenoic acid and odd-chain fatty acids in Schizochytrium sp. S31. Biotechnol Biofuels. 2019;12:141.

13. Guo DS, Ji XJ, Ren LJ, Li GL, Yin FW, Huang H. Development of a real-time bioprocess monitoring method for docosahexaenoic acid production by Schizochytrium sp. Bioresour Technol. 2016;216:422-7.

14. Sun XM, Ren LJ, Ji XJ, Chen SL, Guo DS, Huang H. Adaptive evolution of Schizochytrium sp. by continuous high oxygen stimulations to enhance docosahexaenoic acid synthesis. Bioresour Technol. 2016;211:374-81.

15. Hiltunen JK, Mursula AM, Rottensteiner H, Wierenga RK, Kastaniotis AJ, Gurvitz A. The biochemistry of peroxisomal beta-oxidation in the yeast Saccharomyces cerevisiae. Fems Microbiol Rev. 2003;27:35-64.

16. Xu P, Qiao K, Stephanopoulos G. Engineering oxidative stress defense pathways to build a robust lipid production platform in Yarrowia lipolytica. Biotechnol Bioeng. 2017;114:1521-30.

17. Grimsrud PA, Xie H, Griffin TJ, Bernlohr DA. Oxidative stress and covalent modification of protein with bioactive aldehydes. J Biol Chem. 2008:283:21837-41

18. Li Q, Bai Z, O'Donnell A, Harvey LM, Hoskisson PA, McNeil B. Oxidative stress in fungal fermentation processes: the roles of alternative respiration. Biotechnol Lett. 2011;33:457-67.

19. Mano J, Torii Y, Hayashi S, Takimoto K, Matsui K, Nakamura K, et al. The $\mathrm{NADPH}$ : quinone oxidoreductase P1-zeta-crystallin in Arabidopsis catalyzes the alpha, beta-hydrogenation of 2-alkenals: detoxication of the lipid peroxide-derived reactive aldehydes. Plant Cell Physiol. 2002;43:1445-55.

20. Ruenwai R, Neiss A, Laoteng K, Vongsangnak W, Dalfard AB, Cheevadhanarak $S$, et al. Heterologous production of polyunsaturated fatty acids in Saccharomyces cerevisiae causes a global transcriptional response resulting in reduced proteasomal activity and increased oxidative stress. Biotechnol J. 2011:6:343-56.

21. Lopes M, Mota M, Belo I. Comparison of Yarrowia lipolytica and Pichia pastoris cellular response to different agents of oxidative stress. Appl Biochem Biotechnol. 2013:170:448-58.

22. Jamieson DJ. Oxidative stress responses of the yeast Saccharomyces cerevisiae. Yeast. 1998;14:1511-27.

23. Qin L, Dong S, Yu J, Ning X, Xu K, Zhang SJ, et al. Stress-driven dynamic regulation of multiple tolerance genes improves robustness and productive capacity of Saccharomyces cerevisiae in industrial lignocellulose fermentation. Metab Eng. 2020;61:160-70.

24. Ho KK, Weiner H. Isolation and characterization of an aldehyde dehydrogenase encoded by the aldB gene of Escherichia coli. J Bacteriol. 2005;187:1067-73.

25. Liu B, Liu J, Sun P, Ma X, Jiang Y, Chen F. Sesamol enhances cell growth and the biosynthesis and accumulation of docosahexaenoic acid in the microalga Crypthecodinium cohnii. J Agric Food Chem. 2015;63:5640-5.

26. Xu K, Gao LM, UI Hassan J, Zhao ZP, Li C, Huo YX, et al. Improving the thermo-tolerance of yeast base on the antioxidant defense system. Chem Eng Sci. 2018;175:335-42.
27. Ji XJ, Mo KQ, Ren LJ, Li GL, Huang JZ, Huang H. Genome sequence of Schizochytrium sp. CCTCC M209059, an effective producer of docosahexaenoic acid-rich lipids. Genome Announc. 2015;3:e00819.

28. Kitamura T, Takagi S, Naganuma T, Kihara A. Mouse aldehyde dehydrogenase ALDH3B2 is localized to lipid droplets via two C-terminal tryptophan residues and lipid modification. Biochem J. 2015;465:79-87.

29. Yuzbasheva EY, Mostova EB, Andreeva NI, Yuzbashev TV, Laptev IA, Sobolevskaya TI, et al. Co-expression of glucose-6-phosphate dehydrogenase and acyl-CoA binding protein enhances lipid accumulation in the yeast Yarrowia lipolytica. N Biotechnol. 2017;39:18-21.

30. Chen W, Zhou P, Zhu Y, Xie C, Ma L, Wang X, et al. Improvement in the docosahexaenoic acid production of Schizochytrium sp. S056 by replacement of sea salt. Bioprocess Biosyst Eng. 2016;39:315-21.

31. Sun $X M$, Ren $L$, Bi ZQ, Ji XJ, Zhao QY, Jiang $L$, et al. Development of a cooperative two-factor adaptive-evolution method to enhance lipid production and prevent lipid peroxidation in Schizochytrium sp. Biotechnol Biofuels. 2018;11:65

32. Guo DS, Tong LL, Ji XJ, Ren LJ, Ding QQ. Development of a strategy to improve the stability of culture environment for docosahexaenoic acid fermentation by Schizochytrium sp. Appl Biochem Biotechnol. 2020;192:881-94

33. Liu ZX, You S, Tang BP, Wang B, Sheng S, Wu FA, et al. Inositol as a new enhancer for improving lipid production and accumulation in Schizochytrium sp. SR21. Environ Sci Pollut Res Int. 2019;26:35497-508.

34. Richard D, Kefi K, Barbe U, Bausero P, Visioli F. Polyunsaturated fatty acids as antioxidants. Pharmacol Res. 2008;57:451-5.

35. Guo Z, Olsson L. Physiological response of Saccharomyces cerevisiae to weak acids present in lignocellulosic hydrolysate. FEMS Yeast Res. 2014;14:1234-48.

36. Lamonte G, Tang X, Chen JL, Wu J, Ding CK, Keenan MM, et al. Acidosis induces reprogramming of cellular metabolism to mitigate oxidative stress. Cancer Metab. 2013;1:23.

37. Shen B, Jensen RG, Bohnert HJ. Mannitol protects against oxidation by hydroxyl radicals. Plant Physiol. 1997;115:527-32.

38. Dong Q, Wang Y, Qi S, Gai K, He Q, Wang Y. Histone variant H2A.Z antagonizes the positive effect of the transcriptional activator CPC1 to regulate catalase-3 expression under normal and oxidative stress conditions. Free Radic Biol Med. 2018;121:136-48.

39. Huang J, Xia J, Yang Z, Guan F, Cui D, Guan G, et al. Improved production of a recombinant Rhizomucor miehei lipase expressed in Pichia pastoris and its application for conversion of microalgae oil to biodiesel. Biotechnol Biofuels. 2014;7:111.

40. Ren LJ, Zhuang XY, Chen SL, Ji XJ, Huang H. Introduction of omega-3 desaturase obviously changed the fatty acid profile and sterol content of Schizochytrium sp. J Agr Food Chem. 2015;63:9770-6.

41. Miller GL. Use of dinitrosalicylic acid reagent for determination of reducing sugar. Anal Chem. 1959;31:426-8.

42. Li Z, Yuan H, Yang J, Li B. Optimization of the biomass production of oil algae Chlorella minutissima UTEX2341. Bioresour Technol. 2011;102:9128-34.

43. Ren $L J$, Huang $H$, Xiao AH, Lian M, Jin LJ, Ji XJ. Enhanced docosahexaenoic acid production by reinforcing acetyl-CoA and NADPH supply in Schizochytrium sp. HX-308. Bioprocess Biosyst Eng. 2009;32:837-43.

\section{Publisher's Note}

Springer Nature remains neutral with regard to jurisdictional claims in published maps and institutional affiliations. 